\title{
A Stronger Version of Bogoliubov's Inequality and the Heisenberg Model
}

\author{
G. Roepstorff \\ Institut für Theoretische Physik der TH Aachen, D-5100 Aachen, Federal Republic of Germany
}

\begin{abstract}
On the basis of general inequalities in quantum statistical mechanics we derive a rigorous upper bound for the magnetization in the ferromagnetic quantum Heisenberg model with arbitrary spin and dimension $n \geqq 3$.
\end{abstract}

\section{Introduction}

Recently, Dyson et al. [1] proved the existence of a phase transition at non-zero temperature for the Heisenberg model with nearest neighbor coupling. The proof essentially relies on some new inequalities involving two-point functions. Some of these inequalities are quite general and, therefore, apply to any quantum system in thermal equilibrium. Others rest on the specific structure of the model (spin system, simple cubic lattice, nearest neighbor coupling etc.) and have limited applicability.

Our concern here will be with general estimates of the type used in [1-4]. One of these estimates will turn out to be an improved version of Bogoliubov's inequality [5] which proved to be a powerful tool in many cases. Recall, for instance, that Mermin and Wagner [6] used it to rule out a spontaneous ferromagnetic ordering for the Heisenberg model in one or two dimensions. We shall rederive this result using the stronger inequality and, applying the same argument to three and more dimensions, we shall obtain an upper bound for the reduced magnetization $m$ :

$$
0 \leqq m \leqq M<1 .
$$

In the zero external field limit and for a spin $1 / 2$ lattice the bound $M(\beta)$ is implicitly given by the equation

$$
1-M=2 M(2 \pi)^{-n} \int d^{n} p\left(e^{\beta E_{p} / M}-1\right)^{-1},
$$

where $\beta$ is the inverse temperature and $E_{p}$ stands for the energy of a spin wave with momentum $p$. The integration is carried over the first Brillouin zone. By inspection, the result compares with that of the magnon approximation $[7,8]$ :

$$
1-m=2(2 \pi)^{-n} \int d^{n} p\left(e^{\beta E_{p}}-1\right)^{-1} .
$$


Not only does it respect the upper bound but it also comes close to the upper bound as $m$ approaches 1 at low temperatures.

Since the spin waves describe the low lying excited states where a single spin has deviated from the alignment of the completely ordered state, there are good reasons to believe that at low temperatures the Heisenberg ferromagnet behaves like a system of bosons, called magnons. Since Bloch's approximation (2) neglects the interaction of magnons, over many years a great deal of effort has been focused on calculating deviations predicted by the Heisenberg hamiltonian. The result (1) simply limits the influence of magnon interactions. Notice, however, that the estimate is not good enough to account for the vanishing spontaneous magnetization beyond the critical temperature.

Since $E_{p}$ is of the order $p^{2}$ for small momenta and short range forces, the integral in (1) is infrared divergent unless $n \geqq 3$ or $M=0$. In three or more dimensions, $1-M$ decreases with temperature at $c T^{n / 2}$ with the coefficient given by the spin wave theory.

\section{Some General Inequalities}

Let $\langle\cdot\rangle$ denote the Gibbs state of a finite system with respect to the hamiltonian $H$ and the inverse temperature $\beta$. Given some operator $A$, we may consider various two-point functions associated with $A$ :

$$
\begin{aligned}
& a=\left\langle A^{*} A+A A^{*}\right\rangle / 2 \\
& b=\left\langle A^{*} A-A A^{*}\right\rangle / 2 \\
& c=\beta^{-1}(A, A)=\beta^{-1} \int_{0}^{\beta} d s\left\langle e^{s H} A^{*} e^{-s H} A\right\rangle \\
& d=\beta\left\langle\left[A^{*},[H, A]\right]\right\rangle / 4=\beta\left\langle\left[\left[A^{*}, H\right], A\right]\right\rangle / 4 .
\end{aligned}
$$

In general, these four functions assume independent values. We call explicitly to the reader's attention the fact that these values are real and, apart from $b$, even positive. More restrictions follow from convexity [2] or from the following representation:

There exists a positive measure $d m(x)$ on the real line such that

$$
\begin{aligned}
& a=\int d m(x) \cosh x \\
& b=\int d m(x) \sinh x \\
& c=\int d m(x) x^{-1} \sinh x \\
& d=\int d m(x) x \sinh x .
\end{aligned}
$$

This measure is discrete with bounded support if the system under study is finite. In the thermodynamic limit, provided it exists, the measure may become continuous with unbounded support. We have the symmetry relation $d m(-x)=d m(x)$ if $A=A^{*}$.

The representation (3) is an immediate consequence of Bochner's theorem [9] applied to the function $f(t)=\left\langle A_{t}^{*} A\right\rangle$ where $A_{t}=e^{i t H} A e^{-i t H}$ :

$$
\left\langle A_{t}^{*} A\right\rangle=\int d m(x) \exp (1-2 i t / \beta) x .
$$


The theorem applies since $f$ is positive definite which is easily verified:

$$
\begin{aligned}
\sum_{i, k} \bar{c}_{i} c_{k} f\left(t_{i}-t_{k}\right) & =\left\langle B^{*} B\right\rangle \geqq 0 \\
B & =\sum_{i} c_{i} A_{t_{i}} .
\end{aligned}
$$

The proof carries over to any $\beta$-KMS state since it is invariant under time translations. Moreover, the KMS property implies that

$$
\int d m(x) e^{|x|}<\infty \text {. }
$$

For (3) to exist we must impose a stronger condition:

$$
\int d m(x)|x| e^{|x|}<\infty .
$$

We are now prepared to state the main result of this section.

Theorem. If four quantities $a, b, c$ and $d$ admit a representation of the form (3) where $d m(x)$ is a positive measure on the real line, then the following inequalities hold:

$$
b \operatorname{coth}(b / c) \leqq a \leqq(d c)^{1 / 2} \operatorname{coth}(d / c)^{1 / 2} .
$$

Proof. Upon setting $d \mu(x)=d m(x)(c x)^{-1} \sinh x$ we have that

$$
\begin{aligned}
& a / c=\int d \mu(x) x \operatorname{coth} x \\
& b / c=\int d \mu(x) x \\
& d / c=\int d \mu(x) x^{2} .
\end{aligned}
$$

Now, the function $g(x)=x \operatorname{coth} x$ is convex on the reals and, by construction, the measure $d \mu(x)$ has total mass 1 . From Jensen's inequality [10] we deduce at once that

$$
g\left(\int d \mu(x) x\right) \leqq \int d \mu(x) g(x)
$$

or $(b / c) \operatorname{coth}(b / c) \leqq a / c$. Next, we consider the concave function $G(x)=\sqrt{x} \operatorname{coth} \sqrt{x}$ on the positive reals and have

$$
\int d \mu(x) G\left(x^{2}\right) \leqq G\left(\int d \mu(x) x^{2}\right)
$$

or $a / c \leqq(d / c)^{1 / 2} \operatorname{coth}(d / c)^{1 / 2}$ which completes the proof.

The inequalities (4) already exhaust the information contained in (3) in the sense that they imply any further inequality $F(a, b, c, d) \geqq 0$ which we may deduce on the basis of the representation (3) alone. Indeed, many derived inequalities have special virtues. We therefore list some of them employing the function $F(x)=x \tanh x$ :

$$
\begin{aligned}
& b^{2} \leqq d c \\
& \left(F^{-1}(d / a)\right)^{-2} d \leqq c \leqq\left(\tanh ^{-1}(b / a)\right)^{-1} b, \\
& b \operatorname{coth}(d / b) \leqq a \\
& b \tanh ^{-1}(b / a) \leqq d .
\end{aligned}
$$

Note that (5) follows from (4) since $G^{-1}(x)$ is an increasing function. We obtain (6) unwinding (4), and (8) unwinding (7). In turn, (7) is implied by (4) and (5). 
The lower bound for the Duhamel two-point function $c$ appeared in [1] the upper bound in [2]. Observe that (5) is nothing but Cauchy's inequality applied to the Bogoliubov scalar product $(A,[H, A])$. Bogoliubov's inequality states that $b^{2}$ $\leqq a d$ which was previously derived on the basis of (5) and $c \leqq a$. A stronger version is provided by (7).

It is natural to ask when equality holds in (4)-(8). For this to occur it suffices to assume that $b^{2}=c d$ or, equivalently, that the operators $[H, A]$ and $A$ are linearly dependent. Then $A$ is an eigenvector of the derivation induced by the hamiltonian, i.e. there is some real $E$ (we shall ignore the case $E=0$ ) such that $[H, A]=E A$ and $b / c$ $=\sqrt{d / c}=d / b=\beta E / 2$. It gives us a measure $d m(x)$ which is concentrated at a point. From (4) it may be inferred that

$$
\left\langle A A^{*}\right\rangle=a-b=2 b\left(e^{\beta E}-1\right)^{-1}=2 a\left(e^{\beta E}+1\right)^{-1},
$$

where we used the relations $\tanh x=1-2\left(e^{2 x}+1\right)^{-1}$ and $\operatorname{coth} x=1+2\left(e^{2 x}-1\right)^{-1}$. To see what is involved we shall assume that either $b=1 / 2$ or $a=1 / 2$ (which can be enforced by letting $A^{*} A-A A^{*}=1$ or $A^{*} A+A A^{*}=1$ ). In this case, the thermal average of $A A^{*}$ equals that of a number operator belonging to a Bose or Fermi particle with energy $E$. Think of $A$ as the creation operator for that particle. Then the physical information contained in the equation $[H, A]=E A$ is simply that the particle is free. In essence, the equality sign holds in (4)-(8) if $A$ is a creation or annihilation operator of a free particle.

In other words, the inequalities tell us to what extent the behavior of $A A^{*}$ deviates from that of a number operator if $[H, A]-E A$ is allowed to deviate from zero by a small amount and if $A$ is almost a creation operator.

There are many instances where some operator $A$ obeys "almost" the Bose or Fermi commutation rule in the sense that $b=1 / 2$ or $a=1 / 2$ in some temperature range. A prominent example is the operator $S_{p}^{-} / s^{1 / 2}$ in the context of the Heisenberg model creating a spin wave of momentum $p$ when applied to the ground state. Here we have $b \rightarrow 1 / 2$ as the temperature tends to zero and, therefore, magnons exhibit Bose statistics.

\section{The Héisenberg Model}

The model we are going to consider now constitutes one of the simplest quantum lattice systems with ferromagnetic behavior. The main objective of this section is to introduce our notation and terminology. To start with, we consider a periodic lattice which, abstractly speaking, is a finite (discrete) abelian group $G$ of order $N$. The composition of elements $x \in G$ is written additively and the unit is denoted by 0 . The algebra of observables is identified with the tensor product

$$
\mathfrak{A}=\bigotimes_{x \in G} \mathfrak{A}_{x},
$$

where each $\mathfrak{U}_{x}$ is isomorphic to the full matrix algebra $M_{2 s+1}$ of complex matrices in $2 s+1$ dimensions. The canonical injection $j_{x}: M_{2 s+1} \rightarrow \mathfrak{U}$ identifies observables of a spin $s$ with those of the particular spin at the lattice site $x$. If $S=\left\{S^{2}, S^{2}, S^{3}\right\}$ are the conventional spin operators in $M_{2 s+1}$, we put $S^{i}(x)=j_{x}\left(S^{i}\right)$. 
Let $J(x)$ be a real-valued function on $G$ with the properties $J(x) \geqq 0, J(-x)=J(x)$, and $J(0)=0$. If we think of this function as a measure of the interaction of spins separated by the distance $x$, we are led to the following hamiltonian (with zero energy ground state):

$$
H=\sum_{x, y \in G} J(x-y)\left(s^{2}-S(x) \cdot S(y)\right)
$$

or, in the presence of an external field $h>0$,

$$
H(h)=H+h \sum_{x \in G}\left(s-S^{3}(x)\right) .
$$

In either case the translational invariance of the hamiltonian makes it desirable to reorient the theory with respect to the dual group $\hat{G}$. The duality between $\hat{G}$ and $G$ then supplies the kernel function $\hat{G} \times G \rightarrow T$ of the Fourier transform, $T$ being the unit circle of the complex plane [11]. To be more explicit we recall that any finite abelian group $G$ may be decomposed as $\mathbb{Z}\left(m_{1}\right) \times \ldots \times \mathbb{Z}\left(m_{n}\right), m_{k}>1$, where $\mathbb{Z}(m)$ $=(0,1, \ldots, m-1)$ is the cyclic group of order $m$. In geometrical terms, $m_{k}$ represents the period of the lattice along the $k$ th axis, while $n$ is the dimension and $N=\prod m_{k}$ is the number of sites. Though $\hat{G}$ is isomorphic with $G$, these two groups cannot be naturally identified. Whereas we let $x \in G$ represent the sequence $\left\{x_{k}\right\}$ with $x_{k} \in \mathbb{Z}\left(m_{k}\right)$, it proves very convenient to let $p \in \hat{G}$ represent the sequence $\left\{p_{k}\right\}$ with $m_{k} p_{k} / 2 \pi \in \mathbb{Z}\left(m_{k}\right)$. With this convention, the Fourier kernel is expipx where $p x$ $=\sum p_{k} x_{k}$. In the thermodynamic limit, all periods $m_{k}$ simultaneously tend to infinity yielding $G \cong \mathbb{Z}^{n}$ and $\hat{G} \cong T^{n}$. What will be important for us is that the group mean

$$
N^{-1} \sum_{p \in \hat{G}} f(p)
$$

tends to the normalized Haar measure of the $n$-dimensional torus:

$$
(2 \pi)^{-n} \int_{0 \leqq p_{k} \leqq 2 \pi} d^{n} p f(p) .
$$

In terms of the Fourier transform

$$
S_{p}=N^{-1 / 2} \sum_{x \in G} e^{i p x} S(x)
$$

and the definitions

$$
\begin{aligned}
E_{p} & =\sum_{x \in G} J_{p}(x)=\sum_{x \in G}(1-\cos p x) J(x) \\
E & =N^{-1} \sum_{p \in \widehat{G}} E_{p}=\sum_{x \in G} J(x)
\end{aligned}
$$

we find two relations using Parseval's identity:

$$
\begin{aligned}
N^{-1} \sum_{p \in \widehat{G}} S_{p}^{*} \cdot S_{p} & =N^{-1} \sum_{x \in G} S(x)^{2}=s(s+1), \\
N^{-1} \sum_{p \in \widehat{G}} E_{p} S_{p}^{*} \cdot S_{p} & =N^{-1} H+s E .
\end{aligned}
$$

For later purposes we mention the result of another simple calculation,

$$
\frac{1}{4} \sum_{i}\left[S_{p}^{i *},\left[H, S_{p}^{i}\right]\right]=s^{2} E_{p}-N^{-1} H_{p},
$$


where

$$
H_{p}=\sum_{x, y \in G} J_{p}(x-y)\left(s^{2}-S(x) \cdot S(y)\right) .
$$

Since the coupling is purely ferromagnetic, i.e. $J(x) \geqq 0$, we see that $J_{p}(x) \geqq 0$, hence $H_{p} \geqq 0$ and

$$
\sum_{i}\left[S_{p}^{i *},\left[H, S_{p}^{i}\right]\right] \leqq 4 s^{2} E_{p} .
$$

We conclude this discussion with the remark that $E_{p}$ remains bounded as $N$ tends to infinity, provided that $E<\infty$ which we shall tacitly assume in the sequel. In the limit, $p$ varies continuously and $E_{p} \rightarrow 0$ if $p \rightarrow 0$.

\section{The Magnetization in Thermal Equilibrium}

Let the state vector $\Omega$ describe the ground state of the hamiltonian $H(h)$ which is characterized by the equation $S^{3}(x) \Omega=s \Omega$ and let us introduce the operators

$$
S_{p}^{ \pm}=2^{-1 / 2}\left(S_{p}^{1} \pm i S_{p}^{2}\right) \text {. }
$$

Then $S_{p}^{+} \Omega=0$ and, as is well known, the closed subspaces $\mathscr{H}_{n}$ spanned by the vectors

$$
\Phi_{p_{1}, \ldots, p_{n}}=S_{p_{1}}^{-} \ldots S_{p_{n}}^{-} \Omega, \quad p_{i} \in G
$$

reduce the hamiltonian. $S_{p}^{-}$is viewed to create a magnon with momentum $p$ and $\mathscr{H}_{n}$ is called the $n$-magnon sector. In particular, $H(h) \Phi_{p}=\left(2 s E_{p}+h\right) \Phi_{p}$, so that $2 s E_{p}$ is the kinetic energy and -1 is the magnetic moment of the magnon while $\left(\Phi_{p}, \Phi_{q}\right)$ $=s \delta_{p, q}$. It is helpful to rewrite this as

$$
\left(\Phi_{p}, H(h) \Phi_{p}\right)=\left(\Omega,\left[S_{-p}^{+},\left[H(h), S_{p}^{-}\right]\right] \Omega\right)=s\left(2 s E_{p}+h\right) .
$$

A related result was first proved by Mermin and Wagner [6]: In thermal equilibrium with purely ferromagnetic coupling, the following inequality holds,

$$
0 \leqq\left\langle\left[S_{-p}^{+},\left[H(h), S_{p}^{-}\right]\right]\right\rangle \leqq s\left(2 s E_{p}+h m\right)
$$

if $m$ is the reduced magnetization given by

$$
m=(s N)^{-1} \sum_{x \in G}\left\langle S^{3}(x)\right\rangle=s^{-1}\left\langle S^{3}(0)\right\rangle .
$$

Here and in the following, $\langle\cdot\rangle$ stands for the Gibbs state with respect to the hamiltonian $H(h)$ and the inverse temperature $\beta$. A particular consequence of (18) for $p=0$ is that $m \geqq 0$ since $h>0$ by assumption. Moreover, the log convexity of $\operatorname{tr} \exp (-\beta H(h))$ with respect to $h$ tells us that the magnetization is an increasing function of the external magnetic field as would be expected intuitively.

We shall now prove (18) on the basis of (17). From the Jacobi identity we know that $\left[S_{-p}^{+},\left[H(h), S_{p}^{-}\right]\right]=\left[S_{p}^{-},\left[H(h), S_{-p}^{+}\right]\right]$since $\left[H(h),\left[S_{-p}^{+}, S_{p}^{-}\right]\right]$ $=N^{-1 / 2}\left[H(h), S_{0}^{3}\right]=0$. Now, the automorphism $x \mapsto-x$ of the group $G$ induces an automorphism of the observable algebra $A$ which changes $S_{p}$ into $S_{-p}$ but leaves $H(h)$ invariant. Thus, the Gibbs state is also invariant and we have 
$2\left\langle\left[S_{-p}^{+},\left[H(h), S_{p}^{-}\right]\right]\right\rangle=\left\langle\left[S_{-p}^{+},\left[H(h), S_{p}^{-}\right]\right]+\left[S_{-p}^{-},\left[H(h), S_{p}^{+}\right]\right]\right\rangle$

$=\sum_{i=1,2}\left\langle\left[S_{-p}^{i},\left[H(h), S_{p}^{i}\right]\right]\right\rangle \leqq 2 s\left(2 s E_{p}+h m\right)$ where we used (17) and the positivity of $\left\langle\left[S_{-p}^{3},\left[H(h), S_{p}^{3}\right]\right]\right\rangle$. This completes the proof.

In order to study the implications of (18) we shall now turn our attention to the following two-point functions,

$$
\begin{aligned}
& a_{p}=(1 / 2)\left\langle\left\{S_{-p}^{+}, S_{p}^{-}\right\}\right\rangle \\
& b_{p}=(1 / 2)\left\langle\left[S_{-p}^{+}, S_{p}^{-}\right]\right\rangle \\
& d_{p}=(\beta / 4)\left\langle\left[S_{-p}^{+},\left[H(h), S_{p}^{-}\right]\right]\right\rangle
\end{aligned}
$$

knowing that they are restricted by Bogoliubov's inequality (7):

$$
b_{p} \operatorname{coth}\left(d_{p} / b_{p}\right) \leqq a_{p} .
$$

In fact, $b_{p}$ is independent of the momentum $p$ and related to the magnetization $m$ by the equation $b_{p}=s m / 2$. As (18) provides an upper bound for $d_{p}$ we have

$$
(s m / 2) \operatorname{coth} \beta\left(s E_{p} m^{-1}+h / 2\right) \leqq a_{p} .
$$

If $p=0$ but $h>0$, equality holds in (20) for we know that $\left[H(h), S_{0}^{-}\right]=h S_{0}^{-}$. Looking at our result in the thermodynamic limit, we observe an infrared singularity of the two-point function $a_{p}$ at the point $p=h=0$ provided $m>0$ in some vicinity of this point. This has to be contrasted with the relation (14). We therefore write $\left\langle\left\{S_{-p}^{+}, S_{p}^{-}\right\}\right\rangle=(1 / 2)\left\langle\left\{S_{-p}^{+}, S_{p}^{-}\right\}+\left\{S_{-p}^{-}, S_{p}^{+}\right\}\right\rangle=\sum_{i=1,2}\left\langle S_{-p}^{i} S_{p}^{i}\right\rangle=\left\langle S_{p}^{*} \cdot S_{p}-S_{p}^{3 *} S_{p}^{3}\right\rangle$ where we used invariance of the Gibbs state under momentum reversal. If we know take the mean over the group $\hat{G}$, then

$$
\begin{aligned}
N^{-1} \sum_{p \in \hat{G}} 2 a_{p} & =s(s+1)-N^{-1} \sum_{x \in G}\left\langle S^{3}(x)^{2}\right\rangle \\
& =s(s+1)-\left\langle S^{3}(0)^{2}\right\rangle .
\end{aligned}
$$

To find a useful lower bound for $\left\langle S^{3}(0)^{2}\right\rangle$ we have to exercise some care, for the obvious bound $\left\langle S^{3}(0)\right\rangle^{2}$ gives too little information. Typical best estimates for low $s$ values are

$$
\begin{array}{ll}
s=1 / 2: & \left\langle S^{3}(0)^{2}\right\rangle=1 / 4 \\
s=1: & \left\langle S^{3}(0)^{2}\right\rangle \geqq\left|\left\langle S^{3}(0)\right\rangle\right|
\end{array}
$$

which hold for any state with prescribed value for $\left\langle S^{3}(0)\right\rangle$. To establish a similar result for any spin $s$, we observe first that there is a unique real $j$ such that $j+s$ $=0(\bmod 1)$ and $j \leqq\left|\left\langle S^{3}(0)\right\rangle\right|<j+1$. Then for any state

$$
\left\langle S^{3}(0)^{2}\right\rangle \geqq(2 j+1)\left|\left\langle S^{3}(0)\right\rangle\right|-j(j+1)
$$

which is a simple consequence of the fact that $S^{3}(0)$ possesses the discrete spectrum $\{-s,-s+1, \ldots, s\}$. Reminding us of the defining equation $s m=\left\langle S^{3}(0)\right\rangle>0$ we quickly learn from (20)-(22) that

$$
s(s+1)+j(j+1)-(2 j+1) s m \geqq s m N^{-1} \sum_{p \in \widehat{G}} \operatorname{coth} \beta\left(s E_{p} m^{-1}+h / 2\right)
$$


considering $j$ a function of $m$. While the expression to the right is seen to increase with $m$, the expression to the left turns out to be decreasing. We thus obtain an upper bound $M$ for the magnetization,

$$
0 \leqq m \leqq M<1,
$$

where, in the thermodynamic limit, $M(\beta, h)$ is implicitly defined as the unique solution of the equation

$$
s(s+1)+(j-2 s M)(j+1)=2 s M(2 \pi)^{-n} \int_{\left|p_{k}\right| \leqq \pi} d^{n} p\left(e^{\beta\left(2 s E_{p} M^{-1}+h\right)}-1\right)^{-1}
$$

with $j(M)$ given by $j+s=0(\bmod 1)$ and $j \leqq s M<j+1$.

Suppose now that $j=s-1$, i.e. $1-1 / s \leqq M<1$. This always holds if either $s=1 / 2$ or $s=1$. As for higher spins, it holds at least for sufficiently low temperatures. Our assumption makes the result more intelligible:

$$
1-M=M / s(2 \pi)^{-n} \int_{\left|p_{k}\right| \leqq \pi} d^{n} p\left(e^{\beta\left(2 s E_{p} M^{-1}+h\right)}-1\right)^{-1} .
$$

Observe that the traditional spin wave result is obtained if we replace $M$ by 1 on the right hand side of the Equation (24). As we lower the temperature, $M$ indeed approaches 1 and thus $1-M$ approaches the spin wave result.

Acknowledgement. The author would like to thank E. H. Lieb for communicating the results of Ref. [1] prior to publication.

\section{References}

1. Dyson,F.J., Lieb,E.H., Simon, B.: Phys. Rev. Lett. 37, 120 (1976)

2. Roepstorff,G.: Commun. math. Phys. 46, 253 (1976)

3. Naudts,J., Verbeure, A.: J. Math. Phys. 17, 419 (1976)

4. Szabo, N.: On the phase transition in $X Y$ - and Heisenberg models (preprint)

5. Ruelle,D.: Statistical mechanics. New York: Benjamin 1969

6. Mermin, N., Wagner,H.: Phys. Rev. Letters 17, 1133 (1966)

7. Bloch,F.: Z. Physik 61, 202 (1930)

8. Keffer,F.: Encyclopedia of physics, 18/2. Berlin-Heidelberg-New York: Springer 1966

9. Bochner,S.: Lectures on Fourier integrals. Princeton University Press 1959

10. Rudin,W.: Real and complex analysis. New York: McGraw Hill 1974

11. Loomis, L.H. : An introduction to abstract harmonic analysis. Princeton: Van Nostrand 1953

Communicated by J. L. Lebowitz

Received October 22, 1976

\section{Note Added in Proof}

Prof. F. Dyson kindly informed me that in $1968 \mathrm{H}$. Falk and L. W. Bruch already obtained the "best possible" lower bound (6) for the Duhamel two-point function which they published in Phys. Rev. 180, 442 (1969). 